\title{
Protokoll Mitgliederversammlung der DEGUM
}

Die Mitgliederversammlung hat am Freitag, den 18. Oktober 2019, beim 43. Dreiländertreffen in Leipzig im Congress Center Leipzig, Saal 3, stattgefunden. Anzahl der Teilnehmer: 124

\section{Eröffnung}

Der Präsident, Prof. Dr. med. P. Jecker, begrüßt die Teilnehmer der Mitgliederversammlung.

\section{TOP 1 Annahme der Tagesordnung}

Die Einladung mit Tagesordnung wurde satzungsgemäß auf der Homepage der DEGUM und in der Zeitschrift „Ultraschall in der Medizin“, Heft 4/19, S. 533, veröffentlicht. P. Jecker stellt den Antrag, die Tagesordnung wie veröffentlicht zu bestätigen - weitere Anträge zur Tagesordnung sind dem Vorstand nicht zugegangen.

- TOP 1: Annahme der Tagesordnung

- TOP 2: Annahme des Protokolls der Mitgliederversammlung vom 15.11.2018, Basel

- TOP 3: Rechenschaftsbericht des Vorstands

- TOP 4: Jahresbericht des Schatzmeisters

- TOP 5: Entlastung des Vorstands

- TOP 6: Aufnahme neuer Mitglieder

- TOP 7: EFSUMB: Beschlussfassung über den zukünftigen Mitgliedsstatus der DEGUM (Vollmitgliedschaft vs. Teilmitgliedschaft (Affiliate Membership)) entsprechend der neuen Satzung der EFSUMB

- TOP 8: Antrag des Vorstands zur Neugründung eines Arbeitskreises „Qualitative Ultraschallausbildung“ mit anschließender Beschlussfassung

- TOP 9: Berichte der Sektionen und Arbeitskreise

- TOP 10: Verschiedenes
Die Tagesordnung wird einstimmig angenommen.

\section{TOP 2 Annahme des Protokolls der Mitgliederversammlung beim 42. Dreiländertreffen v. 15.11.18 in Basel}

Das Protokoll war in der Zeitschrift „Ultraschall in der Medizin“ (Heft 1/2019, S. 98101) sowie auf der Homepage der DEGUM veröffentlicht.

Das Protokoll wird ohne Änderungen einstimmig angenommen.

\section{TOP 3 Bericht des Vorstands}

P. Jecker gibt einen Überblick über die Vorstandsaktivitäten des vergangenen Jahres:

Im Januar fand wie üblich außer der Vorstandssitzung auch die Sitzung des Erweiterten Vorstands statt - 3 weitere Vorstandssitzungen folgten in der Geschäftsstelle in Berlin. Im Mai trafen sich die Vorstände von DEGUM, ÖGUM und SGUM zu ihrem jährlichen Austausch in Fulda und ebenfalls im Mai hatte die Akademie ihre Gesellschafterversammlung; der Vertrag mit der Geschäftsführerin Frau Beck wurde verlängert.

Das Thema Strahlenschutzverordnung hat einiges an Aktivitäten gefordert, wie auch der Wechsel des Kongressstandorts des diesjährigen DLTs von Mainz nach Leipzig wegen des Brandes der Rheingoldhalle. Innerhalb von 5 Monaten musste die Planung umgestellt werden - P. Jecker bedankt sich bei den beiden Kongresspräsidenten und allen Beteiligten!
In der neuen Strahlenschutzverordnung, die 2020 in Kraft tritt, steht im $§ 10$ der Satz:

„Bei der Anwendung von Ultraschallgeräten zu nichtmedizinischen Zwecken darf ein Fötus nicht exponiert werden.“

Dieser Satzhat Schwangere verunsichert und bei den Gynäkologen zu Unmut geführt. Es stehen aber auch weitere Fragen im Raum: Darf bei Kursen geschallt werden? Wie ist es auf dem DLT?

Der Vorstand hat mit einer maximalen Öffentlichkeitsarbeit reagiert; eine Pressekonferenz zum Thema in Berlin hat eine gute Resonanz erzielt. Stellungnahmen, Interviews und Beiträge in Funk und Fernsehen wurden von verschiedenen Medien angefragt. Die Herren Heling, Kozlowski, Dudwiesus und Jenderka haben federführend diesen Part übernommen; der Präsident bedankt sich bei allen Akteuren und den 4 Herren besonders.

Weiterhin gab es Kontakte zum Bundesministerium für Gesundheit und zum Bundesministerium für Umwelt und Naturschutz, die für die Strahlenschutzverordnung zuständig sind.

Die neue Gebührenordnung war in den letzten Monaten ebenfalls Thema. Die DEGUM wurde gebeten, Stellung zu nehmen zur Honorierung der Ultraschallleistungen die Legendierung stand bereits fest und war meist über die beteiligten Fachgesellschaften vorgetragen. Zusammen mit Herrn Worlicek hat P. Jecker diese Termine in Berlin in der BÄK wahrgenommen und durch sachliche Argumentation versucht, möglichst gute Ergebnisse für den Ultraschall zu erzielen. 
Die Entscheidung fällen die PKV und das BMG - nähere Einzelheiten werden in der Sitzung des Erweiterten Vorstands im Januar thematisiert.

Eine Zwischenfrage zur Abrechnung von 3D-Ultraschall in der Chirurgie kann nicht beantwortet werden, sondern sollte durch die Fachgesellschaft geklärt werden.

P. Jecker erklärt auf Nachfrage, dass für diese Fragestellung die Bundesärztekammer zuständig ist und nicht die Bundes-KV.

Das DLT 2021 wird von der SGUM organisiert und in Zürich an der ETH stattfinden außer dem Dreiländertreffen wird es dazu einen Kongress für studentische Ausbildung geben. Im Gegensatz zur sonstigen Terminplanung wird dieses Treffen vom 30.06. bis 03.07.2021 stattfinden. In Deutschland haben zu diesem Zeitpunkt bereits einige Bundesländer Sommerferien und für die internen Abläufe in der DEGUM wird zusätzlicher Aufwand nötig. In der Sitzung des Erweiterten Vorstands war das besprochen und entschieden worden, dass es bei einer einmaligen Verschiebung bei einer Teilnahme der DEGUM bleiben sollte. Dieses wurde dem Vorstand im Rahmen der Abendveranstaltung der 3 Vorstände vom SGUM-Präsidenten bestätigt.

In der Forschungsförderung gibt es weiterhin die Entwicklung, dass einige Anträge mit hohen Fördersummen gestellt werden. Zurzeit laufen noch 3 Anträge aus 2018 im Jahr 2019 wurden insgesamt 5 Anträge eingereicht. 1 Antrag ist in der aktuellen Vorstandssitzung positiv beschieden worden, allerdings mit einer geringeren Fördersumme. Bei 2 Anträgen stehen noch Begutachtungen aus und die beiden anderen müssen das Begutachtungsverfahren noch komplett durchlaufen.

P. Jecker bedankt sich bei allen Gutachtern für diese ehrenamtliche Tätigkeit.

Der Wissenschaftspreis 2019 wurde an Frau Dr. med. Katharina Linden aus Bonn verliehen für ihre Arbeit „Left Atrial Volumes and Phasic Function in Healthy Children: Reference Values Using Rael-Time ThreeDimensional Echocardiography“.
Die Preisverleihung erfolgte in diesem Jahr in der Eröffnungsveranstaltung des Kongresses.

Einreichungen für den Promotionspreis gab es keine.

Zukünftig werden die beiden Preise nur noch alle 3 Jahre ausgeschrieben, wenn das Dreiländertreffen in Deutschland stattfindet.

\section{TOP 4 Jahresbericht des Schatzmeisters}

P. Kozlowski stellt den Jahresabschluss 2018 vor, der von der Steuerberatungsgesellschaft Baker Tilly, Berlin, erstellt wurde.

Die steuerliche Bewertung des Jahres 2016 ist noch nicht abgeschlossen, es müssen noch restliche Fragen geklärt werden zum Dreiländertreffen 2016 und der Bewertung zukünftiger Kongresse. Alle Steuerforderungen, die die Jahre ab 2013 betreffen, wurden bereits beglichen. Vonseiten der DEGUM wurde gegen den Bescheid 2016 Einspruch eingelegt.

Der Jahresabschluss 2018 verzeichnet vor Zuführung in die Rücklagen ein Minus von ca. $60000 €$. Die Situation bei den Rücklagen hat sich weiterhin leicht entspannt. Als Verwendung für die zweckgebundenen Rücklagen wird vorgeschlagen: $261642 €$ für die Vereinszeitschrift.

Diesem Vorschlag stimmt die Mitgliederversammlung einstimmig zu.

Das aktuelle Budget für 2019 hält sich bei den Ausgaben für die Forschungsförderung weiterhin auf hohem Niveau und folgt damit den satzungsbedingten Verwendungszwecken. Ebenfalls sind weitere Ausgaben bei der Leitlinienarbeit zu erwarten, die mit $25000 €$ in den Haushalt eingestellt wurden.

Eine Erhöhung wird auch bei den Personalkosten erfolgen, da zum 01.01.19 Frau Strothmann als Assistenz für die Geschäftsführung eingestellt wurde.

Stornierungsgebühren für den abgesagten Kongress in Mainz sind ebenfalls zu berücksichtigen - hier müssen aber die polizeili- chen Ermittlungen und die daraus resultierenden Ergebnisse abgewartet werden.

Entlastung ist ab Mitte 2021 zu erwarten, wenn das Büro in Bonn aufgelöst ist.

Insgesamt ist die finanzielle Situation der DEGUM trotz der erheblichen Steuernachzahlungen sehr solide.

\section{TOP 5 Entlastung des Vorstands}

E. Merz stellt den Antrag auf Entlastung des Vorstands.

Der Antrag wird mit 7 Enthaltungen angenommen.

\section{TOP 6 Aufnahme neuer Mitglieder}

M. Schapheer-Risse präsentiert die aktuellen Zahlen:

Im Jahr 2019 wurden insgesamt 846 Anträge auf Mitgliedschaft in der DEGUM vom Vorstand positiv beschieden.

Die DEGUM hat aktuell 11465 Mitglieder, davon 11337 ordentliche, Kündigungen gab es 475 .

Es sind 3063 Mitglieder mit der Stufe 1 zertifiziert, 1572 mit der Stufe 2, 199 sind als Kursleiter der Stufe II und 508 sind als Kursleiter der Stufe 3 zertifiziert.

M. Schapheer-Risse bedankt sich bei allen Gutachtern und dem ganzen Team der Geschäftsstelle herzlich für die hervorragende Arbeit.

TOP 7 EFSUMB: Beschlussfassung über den zukünftigen Mitgliedsstatus der DEGUM (Vollmitgliedschaft vs. Teilmitgliedschaft (Affiliate Membership)) entsprechend der neuen Satzung der EFSUMB

P. Jecker erläutert kurz die Entwicklung zum Thema seit der letzten Mitgliederversammlung. Der Vorstand hatte als Auftrag erhal- 
ten, im Rahmen der Sitzung des Erweiterten Vorstands die Leitungen der Sektionen und Arbeitskreise über die EFSUMB zu informieren; diese sollten es dann im Laufe des Jahres in ihre Sektionen und Arbeitskreise tragen und ein Stimmungsbild erstellen, damit nun darüber abgestimmt werden kann. Die Sitzung hat im Januar stattgefunden - die informativen Vorträge von C. Jenssen und M. Wüstner, die sie auf der Sitzung gehalten hatten, wurden auf der Homepage der DEGUM im internen Bereich eingestellt. Es waren mehrere Mails zu den aktuellen Entwicklungen verschickt worden - Rückmeldungen gab es von den Sektionen Innere Medizin und Radiologie, der sich die Sektion Chirurgie angeschlossen hat, und eine der Sektion Gynäkologie und Geburtshilfe.

Im Mai wurden anlässlich des EUROSON in Granada u. a. der president elect und der Schatzmeister gewählt. Als president elect wurde Christian Jenssen gewählt und als Schatzmeister Helmut Prosch von der ÖGUM.

Weiterhin wurden J. Künzel, Regensburg, für das Publication Committee und E. v. Bohlen und Halbach, Mainz, für das Students Committee gewählt. K. Jenderka ist bereits Mitglied des Safety Committees und M. Wüstner Mitglied des Education Committees. Somit ist die DEGUM in der EFSUMB gut vertreten.

Außerdem wurde in Granada engagiert über die Neufassung der EFSUMB-Satzung und über die verschiedenen Möglichkeiten der Mitgliedschaft diskutiert. Besprochen und entschieden wurde der zukünftige Mitgliedsbeitrag:

Bei der Vollmitgliedschaft werden zukünftig die zu zahlenden Beiträge für Mitgliedsgesellschaften auf maximal $33 \%$ der Gesamtmitglieder der EFSUMB mit $7 € /$ Mitglied beschränkt. Das bedeutet für die DEGUM, dass der Beitrag wesentlich gesenkt wird. Verbunden ist diese Reduzierung für die DEGUM mit der Aufgabe der absoluten Stimmenmehrheit (Möglichkeit A).

Eine weitere Möglichkeit der Mitgliedschaft ist die Affiliate Membership (Schweizer Modell): Es werden Beiträge nur für die Mitglieder abgeführt, die sich aktiv für eine Mit- gliedschaft in der EFSUMB aussprechen eine Entsendung von Funktionsträgern etc. ist damit nicht mehr möglich (Möglichkeit B).

Bei Austritt einer Ländergesellschaft oder keiner Mitgliedschaft eines Landes gibt es eine individuelle Mitgliedschaft, die $25 € /$ Jahr kostet.

Nach Vorstellung der Möglichkeiten erläutert der Präsident, dass es eine geheime Wahl geben wird - Stimmzettel mit den Möglichkeiten A-C sind vorbereitet, wobei die Möglichkeit C Stimmenthaltung bedeutet (Möglichkeit C).

Es folgt eine kurze Diskussion über eine zu kurze Zeit der Vorbereitung des Themas in den Sektionen sowie der geringen Teilnahme an der Mitgliederversammlung.

Anschließend erfolgt die geheime Abstimmung.

Ergebnis: 113 Mitglieder stimmen für den Vorschlag A, 6 Mitglieder für B, 5 Enthaltungen.

Damit bleibt die DEGUM weiterhin Vollmitglied der EFSUMB und zahlt einen Beitrag von maximal $33 \%$ der gesamten EFSUMBMitglieder.

\section{TOP 8 Antrag des Vorstands zur Neugründung eines Arbeits- kreises „Qualitative Ultraschall- ausbildung" mit anschließen- der Beschlussfassung}

Die DEGUM sieht es als eine ihrer Aufgaben an, die studentische Ausbildung im Ultraschall zu fördern. Bisher sind die Studenten in der AG Studierende in der DEGUM organisiert als assoziierte Mitglieder ohne Stimmrecht. Durch den raschen Wechsel in die Assistenzarztzeit können sich Interessen verlagern.

Engagierte Gruppen an den einzelnen Universitäten sind extrem heterogen und manche entwickeln eine Eigendynamik, der vonseiten der DEGUM gegengesteuert werden sollte.
Auch für die Ausbildung junger Assistenzärzte stehen keine ausreichenden Strukturen innerhalb der DEGUM zur Verfügung es gibt wenig Zeit, ein anspruchsvolles Logbuch, zeitraubende Verwaltungsaufgaben und fehlendes Unterrichten durch erfahrene Ultraschaller.

Dem soll ein neuer Arbeitskreis entgegenwirken: der Arbeitskreis „Qualitative Ultraschallausbildung“; einen entsprechenden Antrag hat P. Jecker an den Vorstand gestellt.

Ziel dieses AKs soll es sein, die Sonografie in der studentischen Ausbildung und für Ärzte in Weiterbildung zu verbessern und zu unterstützen, indem sie durch Ärzte mitgestaltet wird, die über eine ausreichende Expertise sowohl in der Sonografie als auch in der Ausbildung bzw. Lehre verfügen. Die bestehende AG Studierende in der DEGUM soll in diesen Arbeitskreis überführt werden.

Für die Organisation dieses AKs soll vom Vorstand PD Dr. K. Stock aus München vorgeschlagen werden.

Der Antrag auf Gründung dieses Arbeitskreises wurde im Juni gestellt und vom Vorstand einstimmig angenommen.

Ebenso wurde dieser Antrag in der Sitzung des Erweiterten Vorstands am 16.10.19 mehrheitlich angenommen.

In der darauffolgenden sehr engagierten Diskussion werden Zustimmung zur Grundidee des vorgeschlagenen Arbeitskreises wie auch Kritikpunkte vorgetragen:

Die fehlende Kommunikation mit den Studierenden im Vorfeld wird kritisiert. Das Stimmrecht für Studierende muss neu diskutiert werden - bisher haben sie in der Satzung den Status des assoziierten Mitglieds, d. h. sie können in Sektionen und Arbeitskreisen mitarbeiten, haben aber kein Stimmrecht. Der Vorschlag, sie in der aktuellen Sitzung zu ordentlichen Mitgliedern zu machen, kann aus vereinsrechtlichen Gründen nicht umgesetzt werden, da dafür eine Satzungsänderung notwendig ist. 
Weiterhin wird vorgeschlagen, sich nur auf die studentische Ausbildung zu beziehen, da die Weiterbildung Angelegenheit der Kammern ist. Dem widerspricht P. Jecker, da das dem wesentlichen Kern des Arbeitskreises entgegenstünde. Gerade auch die Ausbildung der jungen Assistenten soll mit unterstützt werden.

Insgesamt wird deutlich, dass es mehr Zeit braucht, um dieses Thema weiter vorzubereiten.

P. Jecker bedankt sich bei der Mitgliederversammlung für die lebhafte und gute Dis- kussion und zieht seinen Antrag zurück mit dem Hinweis, das als Aufgabe mit in die nächste Sitzung des Erweiterten Vorstands zu nehmen und im folgenden Jahr erneut in der Mitgliederversammlung zu diskutieren.

\section{TOP 9 Berichte der Sektionen und Arbeitskreise}

Wegen der fortgeschrittenen Zeit wird dieser TOP nicht behandelt.
Die Jahresberichte müssen bis März 2020 in der Geschäftsstelle eingereicht werden.

\section{TOP 10 Verschiedenes}

Keine weiteren Wortmeldungen.

Prof. Dr. med. Peter Jecker

Präsident

Prof. Dr. med. Hans Heynemann

Sekretär

Protokollführerin: Marion Schapheer-Risse 